\title{
Relationship Between Overactive Bladder and Anxiety in Young Men Population in Turkey
}

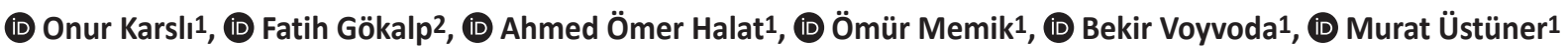 \\ 1 University of Health Sciences Turkey, Kocaeli Derince Training and Research Hospital, Clinic of Urology, Kocaeli, Turkey \\ 2Osmaniye State Hospital, Clinic of Urology, Osmaniye, Turkey
}

\section{Abstract}

Objective: Overactive bladder $(\mathrm{OAB})$ symptoms affect quality of life. The current study evaluates the relationship between anxiety and $0 A B$ in young male patients in Turkish society.

Methods: Male patients aged 18-35 years, diagnosed with OAB between 2015 and 2020, were included in the study. OAB query form (OAB-V8) was filled by participants. Hamilton anxiety scale (HAM-A) was used to evaluate the anxiety levels of OAB patients. OAB-V8 scores were compared statistically before and after anxiolytic treatment of anxious patients.

Results: The mean age of 87 patients was $26.28 \pm 4.53$ (95\% confidence interval: $25.5-27.2)$ and the mean OAB-V8 symptom score was $24.96 \pm 5.7$ (14-36). The OAB-V8 symptom score in the group without anxiety (group 1) and the group with moderate anxiety (group 2) improved significantly at 1 month compared to the pre-treatment period $(p<0.001)$. When the OAB-V8 symptom scores in the third month were analyzed, the $1^{\text {st }}$ group who didn't receive anxiolytic treatment and the $2^{\text {nd }}$ and $3^{\text {rd }}$ group scores receiving anxiolytic treatment were $9.6 \pm 1.8,11.2 \pm 3.2,10.6 \pm 2.8$, and significant change in all three groups compared to the $1^{\text {st }}$ month was observed $(p<0.001)$.

Conclusion: In patients with anxiety, the problems caused by OAB are significantly higher than the normal population. In our study, HAM-A anxiety scores were found higher in patients with $\mathrm{OAB}$. OAB is a common condition that can be seen in the young male population and may accompany anxiety. Treatments combined with multidisciplinary approaches are highly effective in these patients.

Keywords: Overactive bladder, anxiety, male, young

\section{INTRODUCTION}

Overactive bladder $(\mathrm{OAB})$ is a condition characterized by a sudden feeling of urination, accompanied by urinary frequency and nocturia, with incontinence. The frequency of OAB in the USA is $16 \%$ for men and $17 \%$ for women (1). According to the EpiLUTS study conducted in the USA, $27 \%$ of men and $43 \%$ of women experienced symptoms of $\mathrm{OAB}$ at some point in their lives (2).

Studies indicate that many patients with $O A B$ also have nonurological symptoms. It has been reported that symptoms of urination, storage and urine are significantly associated with arthritis, asthma, chronic anxiety, depression, heart disease, irritable bowel syndrome, neurological diseases and sleep disorders (3). OAB symptoms affect daily life, decrease the quality of life, affect work efficiency negatively, disrupt family life, social relationships and sleep patterns (4). Besides all these negativities exacerbate each other, these conditions also negatively affect the mental health of the patients. However, it is still a matter of debate whether anxiety is the cause or the result of $O A B$ $(5,6)$. Studies are generally directed at the fact that OAB impairs the psychosocial situation. However, the number of studies conducted in the literature that the mental state causes OAB is low. Also, most of the studies in the literature are related to female patients. The number of studies on male patients is low. 
The current study evaluates the relationship between anxiety and $\mathrm{OAB}$ in young male patients in Turkish society.

\section{METHODS}

Male patients between the ages of 18-35 who were diagnosed with OAB between 2015 and 2020 were included in this retrospective study. Inclusion criteria; according to the International Continence Association (ICS) 2002 criteria, it was determined that there was a diagnosis of OAB with or without urinary incontinence, infection that could make a feeling of urgent urination, pelvic radiotherapy history, no neurological diseases, and not diagnosed with anxiety and/or treatment in the last 6 months. Clinical evaluation was made according to the AUA guideline with a history, physical examination, urinalysis, post-voiding residual volume. Written consent was obtained from all patients who participated in the study. This study was approved by the University of Health Sciences Turkey, Kocaeli Derince Training and Research Hospital Ethics Committee; 2020/52, and conducted in accordance with the provisions of the Helsinki Declaration.

\section{Evaluation}

The clinical diagnosis of OAB was made using the 2002 ICS diagnostic criteria and published AUA guidelines were followed. All study participants filled the OAB query form (OAB-V8), the Turkish version of which was validated to evaluate lower urinary tract symptoms (7). For the score, 11 was accepted as the threshold value and 2 additional points were added to the score for the male gender.

The patients were consulted to the psychiatry clinic for the diagnosis of anxiety. Hamilton anxiety rating scale (HAM-A) was used to evaluate the anxiety of OAB patients. HAM-A was one of the first grading scales developed to measure the severity of anxiety symptoms and is still widely used in both clinical and research fields today. The scale consists of 14 items, each defined by a series of symptoms and measures both psychic anxiety (mental agitation and psychological distress) and somatic anxiety (physical anxiety-related complaints). Each item is scored on a scale of 0 (unavailable) to 4 (severe), and the total score range is $0-56$. Patients diagnosed with $O A B$ and included in the study were divided into 3 groups according to Hamilton anxiety scores. Those with a score below 17 had no anxiety, 1824 were moderate anxious and greater than 25 were considered severe anxious. The group without anxiety was called group 1 (mild), the group with moderate anxiety (group 2) (group) and the group with severe anxiety group 3 (severe).
Patients diagnosed with $\mathrm{OAB}$ according to the OAB-V8 symptom score were filled with the HAM-A scale before starting anticholinergic treatment. Solifenacin was started at a dose of 5 $\mathrm{mg} /$ day for OAB treatment. OAB-V8 symptom scores were again filled in the patients who were called for control one month later. The 1 month OAB-V8 scores of the patients who were divided into groups according to anxiety severity were compared statistically. Patients who had no improvement in symptom score and had moderate and severe anxiety according to the HAM-A scale before anticholinergic treatment was consulted to the psychiatry department. OAB-V8 symptom scores were reassessed in the $3^{\text {rd }}$ month of patients who were started on anxiolytic treatment appropriate to the patient and continued concomitant anticholinergic treatment by psychiatry. OAB-V8 symptom scores were compared statistically before and after the anxiolytic treatment of anxious patients.

\section{Statistical Analysis}

Statistical Package 23.0 (Social SPSS Statistics; New York, USA) was used for statistical analysis. Data for continuous variables are expressed as mean and standard deviation. Data for categorical variables were expressed as percentage and frequency. For continuous variables, the differences between the three groups analyzed using the Kruskal-Wallis test, and meaningful two groups were analyzed using the Dunnet test. In the OAB score analysis, it was analyzed using Freidman test and Dunn test. Statistical significance level was set at $p<0.05$.

\section{RESULTS}

Ninety-five patients who applied to the outpatient clinic and whose OAB-V8 symptom score was above 11 participated in the study. Three of the patients who participated in the study were excluded from the study because they did not come for control and 5 of them left the drug due to anticholinergic side effects. The mean age of 87 patients participating in the study was 26.28 \pm 4.53 (95\% confidence interval: $25.5-27.2$ ) years, and the patients' OAB-V8 symptom score was $24.96 \pm 5.7$ (14-36). Before anticholinergic treatment, HAM-A scale was completed. Mild anxiety was detected in 49 patients, moderate in 22 patients, severe anxiety in 16 patients and patients were divided into 3 groups accordingly. The median OAB-V8 symptom score before treatment was found as 20 [interquartile range (IQR): 18.0-26.0], 28 (IQR: 26.0-30.0), 31 (IQR: 28.0-33.0) in groups 1, 2, and 3 respectively. OAB-V8 score before treatment was significantly lower in group $1(p<0.001)$, but there was no significant difference between groups 2 and $3(p=0.22)$. A significant improvement was achieved in the OAB-V8 symptom score at 1 
month compared to the pre-treatment period in the group of patients without anxiety (group 1) and group with moderate anxiety (group 2) $(p<0.001)$ (Figure 1). However, in the group with severe anxiety (group 3), it was observed that there was a statistically insignificant decrease in the OAB score at 1 month $(p=0.64)$ (Table 1).

When the OAB-V8 symptom scores in the third month were analyzed, the $1^{\text {st }}$ group who did not receive anxiolytic treatment and the $2^{\text {nd }}$ and $3^{\text {rd }}$ group scores receiving anxiolytic treatment was 10 (IQR: 9.0-10.0), 10 (IQR: 9.0-13.0), 10.5 (IQR: 9.0-11.5), and the significant change was observed in all three groups at $3^{\text {rd }}$ month compared to the pretreatment $(p<0.001)$. There was no statically considerable change found between $1^{\text {st }}$ month and $3^{\text {rd }}$ months scores $(p=0.10)$. Additionally, there was no statistically significant found between 1st month and pretreatment scores in group $3(p=0.64)$.

\section{DISCUSSION}

In a study conducted by the world health organization, anxiety was reported to be the most common psychiatric disorder

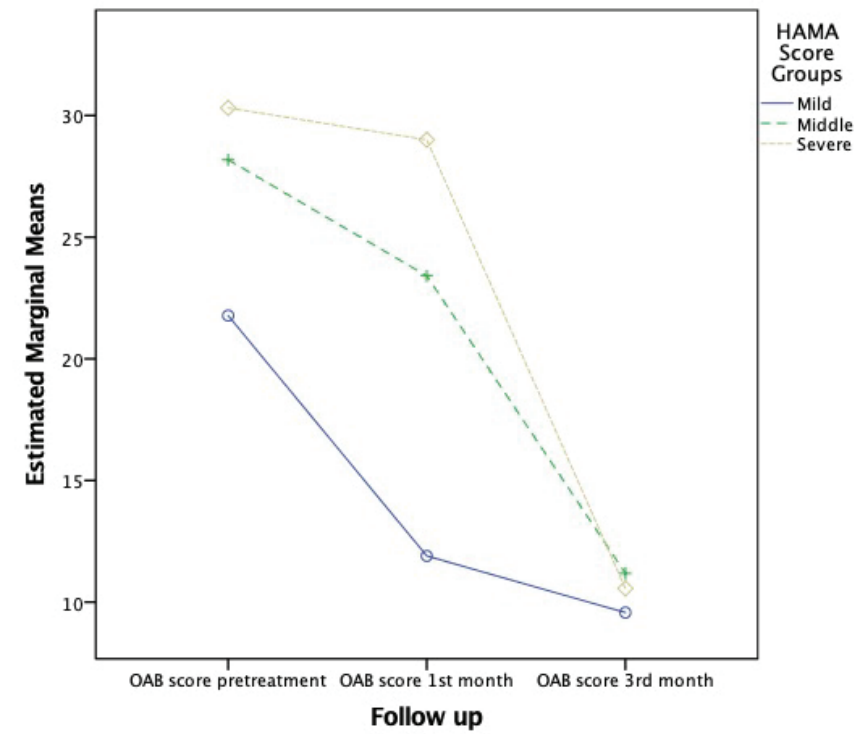

Figure 1. OAB-V8 score changes of groups after treatment OAB: Over active bladder, HAM-A: Hamilton anxiety scale globally (8). The prevalence of lifelong anxiety disorder in the USA is around $18 \%$. Some researchers thought that psychosomatic disorders such as anxiety may affect the pathogenesis of $O A B(9)$, and this has been shown in clinical-based and epidemiological studies (10). In our study, a relationship was found between $\mathrm{OAB}$ and anxiety. The relationship between affective disorders and lower urinary tract symptoms was first described by Engel in 1964. The link between the brain and bladder has not been clarified today, but it has been reported that it may be using the same neuropharmacological pathways according to the hypotheses $(10,11)$. It is unclear which $\mathrm{OAB}$ and anxiety that are thought to be related trigger the other.

In a study by Milsom et al. (12), $O A B$, and $O A B$ in participants with hospital anxiety and depression scale (HADS) -anxiety and HADS-depression (HADS-D) scores and who showed clinically significant anxiety and depression levels. The problems caused by the patients were found to be significantly higher than those with low anxiety and depression scores $(p<0.001)$. In logistic regression analysis, the strongest ailment markers associated with $O A B$ for both men and women were determined as urine urgency, urinary incontinence, urinary frequency and nocturia, respectively. A significant increase in $\mathrm{OAB}$-related disorders were observed in male participants as the level of education, HADSanxiety (HADS-A) score and general health problems increased. In our study, as the level of anxiety increases in male patients, the OAB-V8 symptom score increases and the treatment of $O A B$ becomes difficult. With anxiolytic treatment given in addition to anticholinergic treatment, a significant improvement in OAB-V8 symptom score is observed in the $3^{\text {rd }}$ month. In the EpiLUTS study, in the interviews with patients with $O A B$ symptoms, the reason for the anxiety of the patients was stated as the concern of finding a toilet in time and the urinary incontinence. Simultaneously, constant anxiety of going to the toilet in some participants led to a feeling of hopelessness and depression in patients (12).

In the literature, Lai et al. (13) in a study he conducted and compared the psychological scales, $O A B$ patients with common systemic symptoms had worse HADS-D, HADS-A,

Table 1. OAB-V8 scores of anxiety groups

\begin{tabular}{|c|c|c|c|c|}
\hline & \multicolumn{4}{|c|}{ HAM-A score groups } \\
\hline & Mild & Moderate & Severe & $\mathbf{p}$ \\
\hline OAB score pretreatment & $20(18.0-26.0)$ & $28(26.0-30.0)$ & $31(28.0-33.0)$ & $p<0.001$ \\
\hline OAB score $3^{\text {st }}$ month & $10(9.0-10.0)$ & $10(9.0-13.0)$ & $10.5(9.0-11.5)$ & $p=0.16$ \\
\hline
\end{tabular}


fatigue symptoms (PROMIS-Fatigue) and higher than $\mathrm{OAB}$ patients without common systemic symptoms. They detected psychological stress (13). Results were statistically significant for all comparisons adjusted for age and gender $(p<0.05)$. Similar to the literature, HAM-A scores were higher in patients with $\mathrm{OAB}$ with urinary incontinence in our study.

In a study of Alves et al. (14), Hopkins investigated the relationship of OAB with anxiety symptoms using three items of the symptom checklist, and although the cause-effect relationship could not be established, the authors concluded that individuals with $\mathrm{OAB}$ are more prone to anxiety symptoms. Similar to these results, although we showed the relationship between anxiety and $\mathrm{OAB}$ in our study, we could not show the cause-effect relationship between the two disorders.

\section{CONCLUSION}

$\mathrm{OAB}$ is a common condition that can also be seen in the young male population and may accompany anxiety. Questioning the psychosomatic complaints of patients and treating them with a multidisciplinary approach may provide better results.

\section{Ethics}

Ethics Committee Approval: This study was approved by the University of Health Sciences Turkey, Kocaeli Derince Training and Research Hospital Ethics Committee; 2020/52, and conducted in accordance with the provisions of the Helsinki Declaration.

Informed Consent: Written consent was obtained from all patients who participated in the study.

Peer-review: Externally and internally peer-reviewed.

\section{Authorship Contributions}

Concept: O.K., B.V., Design: O.K., B.V., Data Collection or Processing: O.K., M.Ü., Analysis or Interpretation: F.G., Literature Search: A.Ö.H., Writing: O.K., Ö.M.

Conflict of Interest: No conflict of interest was declared by the authors.

Financial Disclosure: The authors declared that this study received no financial support.

\section{REFERENCES}

1. Stewart WF, Van Rooyen JB, Cundiff GW, Abrams P, Herzog AR, Corey R, et al. Prevalence and burden of overactive bladder in the United States. World J Urol 2003;20:327-36.

2. Coyne KS, Sexton CC, Vats V, Thompson C, Kopp ZS, Milsom I. National community prevalence of overactive bladder in the United States stratified by sex and age. Urology 2011;77:1081-7.

3. Coyne KS, Kaplan SA, Chapple CR, Sexton CC, Kopp ZS, Bush EN, et al. Risk factors and comorbid conditions associated with lower urinary tract symptoms: EpiLUTS. BJU Int 2009;103(Suppl 3):24-32.

4. Coyne KS, Sexton CC, Irwin DE, Kopp ZS, Kelleher CJ, Milsom I. The impact of overactive bladder, incontinence and other lower urinary tract symptoms on quality of life, work productivity, sexuality and emotional well-being in men and women: results from the EPIC study. BJU Int 2008;101:1388-95

5. Bogner HR, Gallo JJ, Swartz KL, Ford DE. Anxiety disorders and disability secondary to urinary incontinence among adults over age 50. Int J Psychiatry Med 2002;32:141-54.

6. Felde G, Bjelland I, Hunskaar S. Anxiety and depression associated with incontinence in middle-aged women: a large Norwegian cross-sectional study. Int Urogynecol J 2012;23:299-306.

7. Tarcan T, Mangır N, Özgür MÖ, Akbal C. OAB-V8 Overactive bladder questionnaire validation study. Üroloji Bülteni 2012;21:113-6.

8. Wang PS, Lane M, Olfson M, Pincus HA, Wells KB, Kessler RC. Twelvemonth use of mental health services in the United States: results from the National Comorbidity Survey Replication. Arch Gen Psychiatry 2005;62:629-40.

9. Freeman RM, McPherson FM, Baxby K. Psychological features of women with idiopathic detrusor instability. Urol Int 1985;40:257-9.

10. Zorn BH, Montgomery H, Pieper K, Gray M, Steers WD. Urinary incontinence and depression. J Urol 1999;162:82-4.

11. Klausner AP, Steers WD. Corticotropin releasing factor: a mediator of emotional influences on bladder function. J Urol 2004;172:2570-3.

12. Milsom I, Kaplan SA, Coyne KS, Sexton CC, Kopp ZS. Effect of bothersome overactive bladder symptoms on health-related quality of life, anxiety, depression, and treatment seeking in the United States: results from EpiLUTS. Urology 2012;80:90-6.

13. Lai HH, Vetter J, Jain S, Andriole GL. Systemic nonurological symptoms in patients with overactive bladder. J Urol 2016;196:467-72.

14. Alves AT, Jácomo RH, Gomide LB, Garcia PA, Bontempo AP, Karnikoskwi MG. Relationship between anxiety and overactive bladder syndrome in older women. Rev Bras Ginecol Obstet 2014;36:310-4. 